\title{
El estudio de la comunicación publicitaria online en el Grado en Publicidad y RR.PP.
}

Araceli Castelló Martínez I araceli.castello@ua.es

UNIVERSIDAD DE ALICANTE

Resumen: Las acciones de comunicación corporativa y publicitaria a través de los medios digitales se han convertido en una parte esencial en las estrategias de comunicación de la empresa y la marca. La presente investigación se centra en estudiar la inclusión de asignaturas vinculadas con el estudio del marketing digital y la publicidad online en los planes de estudio del grado en Publicidad y RR.PP., con el fin de ver su adaptación a una realidad profesional cambiante que cada vez más requiere de comunicadores formados en el ámbito de la comunicación digital.

Palabras clave: Espacio Europeo de Educación Superior, Ciencias de la Comunicación, Publicidad, Internet

Abstract: Corporate and advertising communication through digital media have become an essential part in company and brand communication strategies. The present research focuses on studying the inclusion of subjects related to the study of digital marketing and online advertising in the curriculum of the degree in advertising and public relations, in order to see its adaptation to a changing professional reality that increasingly requires communicators trained in the field of digital communication.

Key words: European Higher Education Area, Communication, studies, Advertising, Internet 


\section{Introducción}

El crecimiento experimentado por la publicidad interactiva en los últimos años ha llevado a Internet a ser uno de los medios más populares, si no el que más, entre las estrategias de comunicación corporativa y publicitaria de los anunciantes. Desde el punto de vista de la comunicación y la planificación publicitarias, las opciones que ofrece Internet han convertido a agencias de publicidad y agencias de medios en prescriptoras del medio online, pasando a ser vital, por tanto, la especialización y la profesionalización del sector en este ámbito.

De esta manera, cada vez más se requiere de profesionales expertos en comunicación publicitaria online. Con la popularización de las plataformas 2.0 (Facebook, Twitter, LinkedIn, Youtube, etc.) y el interés de las empresas en tener presencia en ellas, el perfil profesional de moda en el mercado publicitario es el del Community Manager, como el gestor de las comunidades online de la empresa.

La preparación de los universitarios estudiantes del Grado en Publicidad y RR.PP., dentro de las bases sentadas por el Espacio Europeo de Educación Superior (EEES) a propósito de la formación en aquellas competencias que les serán demandadas cuando se integren en el ámbito profesional, obliga a una revisión continua de las tendencias en el sector profesional a la hora de elaborar los planes de estudio del Grado. Qué presencia tiene el estudio de la comunicación publicitaria online desde el punto de vista de la estrategia y la planificación es la pregunta a la que trata de responder este estudio.

\section{Metodología}

El presente estudio trata de dar respuesta a dos hipótesis iniciales, paralelas al crecimiento experimentado por Internet como medio de comunicación y, principalmente, soporte publicitario en cada una de sus manifestaciones (mobile marketing, e-commerce, e-mailing, marketing de afiliación, displays, etc.), especialmente en lo que a Social Media Marketing y Community Management se refiere:

- La amplia oferta de formación superior (posgrados y másters) especializada en marketing digital e interactivo existente en el curso académico 2010-2011.

- La escasa presencia de módulos y/o asignaturas en los planes de estudio del Grado en Publicidad y RR.PP. centradas en el estudio de la comunicación publicitaria online.

Para la elaboración del marco teórico se lleva a cabo una investigación documental, identificando aquellas publicaciones y estudios, tanto académicos como profesionales, relacionados con la materia tratada y publicados en los últimos cinco años.

El trabajo de campo se basa, por una parte y para la verificación de la primera hipótesis, en el sondeo de la oferta formativa tanto de escuelas de negocios como de universidades 
públicas y privadas. Para ello se realiza una búsqueda de las palabras clave "curso redes sociales" y "curso community manager" y se selecciona una muestra representativa de la oferta de formación superior existente en España en el curso 2010-2011.

Por otra parte, la segunda hipótesis se estudia mediante la revisión de los planes de estudio de todos los Grados en Publicidad y RR.PP. de universidades españolas, identificando la presencia de módulos y/o asignaturas cuya denominación se refiriera a la publicidad, el marketing y/o la comunicación digital. Puesto que el objeto de investigación son los estudios con esta temática desde el punto de vista de la estrategia de la comunicación y/o la planificación publicitaria, se descartan aquellos módulos y/o asignaturas que, de acuerdo con su definición, se refieren al estudio del medio online desde el punto de vista del diseño, la tecnología y/o la informática, como por ejemplo "Tecnologías digitales aplicadas a la publicidad", "Diseño publicitario digital", "Gestión de contenidos digitales" o "Usos e impactos de las TIC".

En total, se analiza el plan de estudios para el Grado en Publicidad y RR.PP. de 40 universidades en el curso 2010-2011. En aquellas universidades que no cuentan con un Grado en Publicidad y RR.PP., se observa si cuentan, en su defecto, con Grados en Comunicación y/o Marketing, y se analiza el plan de estudios de esta oferta formativa, por entender la publicidad al servicio de los objetivos de comunicación y, en un nivel más amplio, de los objetivos de marketing.

El trabajo de campo se realizó durante los meses de abril y mayo de 2011, por lo que el estudio cuenta con limitaciones desde el punto de vista metodológico al mostrar los resultados para un momento determinado. Estas limitaciones temporales obligan a una revisión de los resultados en posteriores oleadas, conforme vaya incrementándose la oferta formativa en la materia y se vayan implementando los Grados de Publicidad y RR.PP. en las Universidades Españolas ${ }^{1}$.

\section{EI EEES y el Grado en Publicidad y RR.PP.}

Desde que en 1987 se impulsara el programa de movilidad Erasmus hasta la Declaración de Bolonia de 1999 y su posterior implantación progresiva en España y el resto de países del Espacio Europeo de Educación Superior (EEES), entre 2008 y 2010, con el objetivo de armonizar los sistemas universitarios europeos, además de la movilidad e intercambio de alumnos y la estructuración de las titulaciones y las materias en créditos europeos a través del European Credit Transfer System (ECTS) ${ }^{2}$, el aspecto principal en el nuevo modelo

\footnotetext{
1 Un ejemplo de la nueva oferta formativa lo encontramos en la Universidad de Alicante, que ha pasado de no tener estudios de posgrado sobre marketing digital en el curso 2009-2010 a contar con varios títulos propios en el curso 2010-2011, especializados en medios sociales, reputación online y community management.

2 El Real Decreto 1125/2003 establece, entre otras cuestiones, la definición y la valoración del crédito europeo. El crédito europeo tendrá una valoración de entre 25 y 30 horas contando las horas de clases teóricas y prácticas, las horas de estudio, las dedicadas a seminarios y trabajos y las horas de preparación y realización de examen.
} 
pedagógico ha sido la formación basada en competencias del estudiante, lo que ha implicado "una transformación radical en los planteamientos didácticos" (Pérez Serrano et al, 2010: 2).

La formación basada en competencias, que busca capacitar al alumno para ejercer con éxito la actividad profesional para la que cualifica el grado, destaca, en este sentido, "el acercamiento entre la universidad y la realidad empresarial", apostando "por una formación de tipo más práctica y por la resolución de problemas simulando una situación real del mercado laboral" (Sierra Sánchez y Cabezuelo Lorenzo, 2010: 32).

El Real Decreto 1393/2007, de 29 de octubre, es el que establece la ordenación de las enseñanzas universitarias oficiales y desarrolla la estructura y el diseño de los estudios de Grado, Máster y Doctorado, con un plazo límite para la puesta en marcha de estas medidas en 2010. El título de Grado es el primer nivel en la nueva estructura del sistema universitario. Proporciona al estudiante una formación general en una disciplina determinada con el objeto de prepararlo para el mundo del trabajo. Asimismo, lo capacita para acceder al segundo nivel de las enseñanzas universitarias, el Máster.

En los títulos de Grado existen tres tipos de asignaturas: formación básica, obligatorias y optativas. Las asignaturas de formación básica, impartidas en los dos primeros cursos, son de carácter general dentro de una rama de conocimiento y no necesariamente específicas de la titulación. Por su parte, las asignaturas obligatorias son el contenido específico de la titulación, ya que responden a las competencias específicas de ese título, por lo que deben ser cursadas por todos los estudiantes. Por último, las optativas son aquellas asignaturas elegidas por los alumnos entre la oferta puesta a disposición de la Universidad.

Con respecto a los estudios de Grado en Comunicación, el programa formativo planteado por cada Universidad sigue la guía del Libro Blanco publicado por la Agencia Nacional de Evaluación de la Calidad y la Acreditación (ANECA), según la cual el reparto de contenidos del Título de Publicidad y RR.PP. debe contener un $50 \%$ de contenidos comunes obligatorios, un $10 \%$ de contenidos instrumentales obligatorios y un $40 \%$ de contenidos propios de la Universidad (ANECA, 2005: 333).

El Libro Blanco también destaca la formación del alumno en competencias disciplinares (saber), profesionales (saber hacer), académicas y específicas (2005: 272). Entre los conocimientos disciplinares se incluyen el "estudio de las estrategias y procesos encaminados a la creación y realización de mensajes publicitarios en los distintos soportes y medios de comunicación" y el "conocimiento de la estructura de los medios publicitarios: características, tipologías y problemáticas" (2005, 273-274). Igualmente, dentro de las competencias profesionales destacan, por su vinculación con el tema tratado en el presente artículo, la "capacidad y habilidad para establecer el plan de comunicación (fijar objetivos de comunicación, definir el público objetivo, plantear estrategias y controlar el presupuesto de comunicación)", así como la "capacidad y habilidad para utilizar las tecnologías y técnicas comunicativas en los distintos medios o sistemas mediáticos combinados e interactivos 
(multimedia), especialmente para aplicarlas al mundo de la comunicación, con especial énfasis en la creación de nuevos soportes".

Este último conocimiento es, de media, la competencia profesional más valorada por los profesionales de la comunicación para los distintos perfiles definidos por ANECA: director/a de comunicación, investigador/a y consultor/a estratégico en Publicidad y RR.PP.; investigadores/as, planificadores/as y compradores de medios; creativo/a y diseñador/a y gestor/a de comunicación corporativa (2005:270-272).

Por la variedad de especializaciones existentes en el mundo profesional y en el mercado laboral del marketing, la comunicación y la publicidad, la titulación de Publicidad y RR.PP. se caracteriza por tener los perfiles profesionales más diferenciados entre sí.

El Libro Blanco apunta como objetivo fundamental del Grado en Publicidad y RR.PP. que "Ios estudiantes de Publicidad y Relaciones Públicas han de adquirir un conocimiento básico y general de los principales puntos de inflexión que configuran el panorama actual de la labor del profesional de la comunicación, especialmente publicitaria y de las relaciones públicas" (2005: 329). Para ello, la formación debería basarse, entre otros, en los siguientes saberes y habilidades (2005: 330-331):

\footnotetext{
Formación en las capacidades expresivas y particularidades de cada uno de los medios, soportes y formatos publicitarios para la elaboración de mensajes y campañas de comunicación, así como en la habilidad para analizar y seleccionar los vehículos de difusión de acuerdo con las estrategias de comunicación (tanto en medios convencionales como en medios no convencionales.

Formación en el conocimiento y uso de las tecnologías de la comunicación en los distintos entornos multimedia e hipermedia, para su aplicación en el ámbito de la publicidad y las relaciones públicas y el desarrollo de nuevos soportes.
}

El estudio centrado en las habilidades que en el futuro serán demandadas a los alumnos una vez finalicen sus estudios en el ámbito profesional favorece "una mayor dosis de motivación del alumnado ya que, si son conscientes que lo que realizan dentro del aula es muy similar a lo que les espera fuera de ella, el grado de implicación tiende a ser mayor" (Fanjul y González, 2010). Es por esto por lo que el EEES valora mucho más el autoaprendizaje, el trabajo autónomo y el uso de las tecnologías de la información y de la comunicación, fomentando el "aprender a aprender" por parte del alumno.

\section{La comunicación publicitaria online}

La Red es hoy en día una de las herramientas fundamentales en la interacción y la comunicación de personas, empresas e instituciones. De acuerdo con la tercera oleada de 2011 del "Estudio General de Medios" publicado por la Asociación para la Investigación de Medios de Comunicación (AIMC), la audiencia mensual de Internet asciende al 57,6\% de la población española de 14 años o más y el $75 \%$ de los internautas accede a la Red a diario.

Además, tras televisión y prensa, Internet es el tercer medio en inversión publicitaria, representando el $15,62 \%$ del total para el primer semestre de 2011 , y el único que ha 
crecido de manera continua desde 2005, según el "Estudio InfoAdex de la inversión publicitaria en España", con incrementos del 20,7\% de 2009 a 2010 y del 14,4\% del primer semestre de 2010 al mismo periodo de 2010.

De esta manera, Internet se ha convertido para muchas empresas en el medio idóneo para personalizar su mensaje, interactuar con el destinatario y generar nuevas oportunidades de negocio. Afinidad con el público objetivo, generación de notoriedad con costes reducidos, segmentación y medición en tiempo real son algunas de las ventajas del medio más alabadas por los anunciantes. En palabras de Madinabeitia (2010: 43), "la digitalización de la sociedad ha traído consigo grandes cambios en todos los ámbitos, siendo el mundo de la comunicación y en concreto el de la comunicación comercial uno de los más afectados".

La Web 2.0 ha generado toda una revolución en el ámbito de la publicidad, en particular, y la comunicación empresarial, en general. En los Social Media, el consumidor se coloca en el centro de la relación con las marcas y, ahora más que nunca, de él depende el éxito empresarial. Como afirma Bermejo (2008: 49), "asistimos al inicio de la interactividad, así como a la formación de redes y comunidades interrelacionadas donde el receptor además de audiencia, de público y de consumidor, deviene usuario y prosumer, es decir, co-productor, distribuidor y consumidor, todo al mismo tiempo". Esta capacidad de "generar conversaciones" ha implicado un cambio radical en la comunicación comercial (Madinabeitia, 2010: 49).

Según datos de la empresa de medición de audiencia online ComScore, a finales de 2010 hay más de 945 millones de usuarios de redes sociales en el mundo, 174 de ellos en Estados Unidos. Facebook es la red social por antonomasia, superando los 500 millones de usuarios. En España, de acuerdo con la edición de 2011 del estudio anual "Navegantes en la Red" publicado por AIMC, las redes sociales son un fenómeno en continuo ascenso: un 60,3\% de la población internauta confirma que estas plataformas no son una moda pasajera, ya que se conecta a ellas diariamente, frente al $43,5 \%$ que lo hacía en 2009 y el $28,6 \%$ que accedía diariamente en 2008.

Facebook es la red social más popular en nuestro país, al conectarse a ella el $90 \%$ de los usuarios de redes sociales, seguida de Tuenti y Twitter. En el caso de esta última, destaca el crecimiento experimentado de 2009 a 2010 , de $12,7 \%$ a $21,9 \%$. Es por esto por lo que "las redes son un nuevo y atractivo canal que las marcas desean utilizar para reactivar su alicaído branding" (Campos, 2008: 291).

La presencia de empresas y marcas en las plataformas 2.0 es una realidad motivada por las bondades de las plataformas sociales en el ámbito de la comunicación empresarial y publicitaria. Los nuevos entornos digitales (redes sociales, blogs, plataformas de videos digitales, microblogging, la geolocalización al servicio del mobile marketing, etc.) favorecen la orientación empresarial basada en las relaciones (la filosofía del client first), la generación de branding social, la segmentación y personalización de mensajes, la evangelización de la 
marca a través de la prescripción y la viralidad y la puesta en marcha de un experiential marketing que genere customer engagement (Castelló Martínez, 2010: 79-98).

Según Celaya (2008: 146-147), "el principal retorno que buscan las empresas a la hora de invertir en tecnologías Web 2.0 es la mejora de la interacción con sus clientes e incrementar sus ventas". Por su parte, Ros (2008: 24) señala que "con estas nuevas herramientas de participación, gestionar conocimiento para generar valor al público de interés de la organización debe ser el objetivo".

El informe "Marketing en medios sociales" publicado por Territorio Creativo en abril de 2010 destaca que 8 de cada 10 empresas que llevan a cabo acciones de Social Media Marketing aprueban la eficacia de los medios sociales para generar notoriedad de marca. Del lado del consumidor, la edición de 2010 del estudio "The socialisation of Brands", realizado por la agencia de medios Universal McCann resalta cómo al haber pasado a formar parte de una comunidad de marca, el $72 \%$ de los encuestados declaraba tener una visión más positiva de la marca, el 71\% reconocía tener más probabilidad de comprar la marca, el $66 \%$ se sentía más fiel a ella y el $63 \%$ había invitado a otros usuarios a unirse a la comunidad.

En definitiva, junto con el amplio abanico de tácticas y herramientas que ofrece el medio Internet (displays, SEO y SEM, e-mailing, e-commerce, marketing de afiliación, mobile marketing), entornos colaborativos y tecnologías participativas como Facebook y Twitter han convertido a las acciones online en parte esencial en la estrategia de comunicación de empresa, producto y marca.

\section{Un nuevo perfil profesional: el Community Manager}

La rápida evolución del entorno digital, ya mucho antes de la llamada Web 2.0, había supuesto la continua aparición de nuevos roles profesionales, como expertos en Search Engine Optimization (SEO) y Search Engine Marketing (SEM), maquetadores expertos en lenguaje HTML, diseñadores con conocimientos de nuevos programas, planificadores y compradores de espacios expertos en el medio online, etc. El crecimiento de los Social Media y el interés por parte de las empresas en tener presencia corporativa y publicitaria en las plataformas 2.0 han venido acompañados de la demanda de un nuevo profesional: el Community Manager.

Según el estudio "Tendencias del mercado laboral en el sector de la publicidad digital", elaborado por Interactive Advertising Bureau Spain (IAB Spain), la proyección de futuro de su puesto de trabajo es uno de los puntos fuertes para los profesionales del mercado de la publicidad digital. Si tenemos en cuenta que las principales temáticas sobre las que les gustaría actualizar conocimientos y competencias son el Social Media Optimization (SMO) y el Social Media Marketing (SMM), las redes sociales, la Web 2.0 y 3.0 o el Cloud Computing y que estas áreas van a ser, en opinión de los profesionales del mercado de la publicidad 
digital, las más demandadas a corto-medio plazo, parece más que evidente cuál es la tendencia que está viviendo el sector.

Con la revolución de los Social Media, en opinión de los profesionales del mercado de la publicidad digital, el Community Manager va a ser el perfil más demandado por los anunciantes en los próximos años, seguido de comerciales y expertos en Social Media, redes sociales y Web 2.0, según el estudio de IAB Spain:

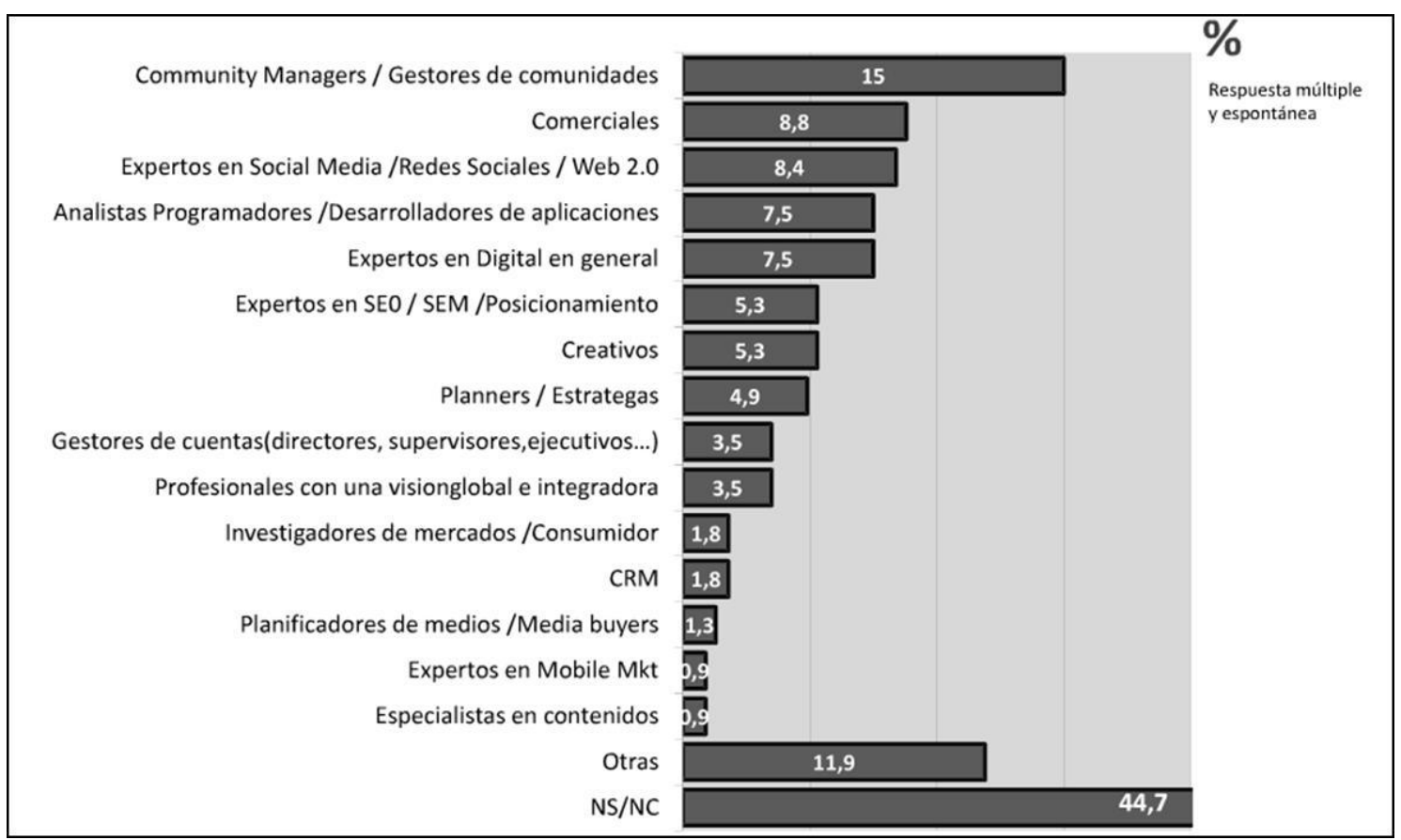

Cuadro 1. Perfiles más demandados

En Infojobs (www.infojobs.net), portal de Internet líder en España en ofertas de empleo, con las palabras clave "Community Manager" se encontraron el 26 de abril de 2011 más de cuarenta ofertas, todas ellas vinculadas con la comunicación online. En la siguiente tabla hemos incluido las más afines a este perfil: 


\begin{tabular}{|c|c|c|c|c|c|c|}
\hline & Puesto vacante & Provincia & Categoría & Mivel de e studios requerido & Experiencia minima & Salario \\
\hline 1 & Community Manager & BCN & MK y Com - Desarrollo de marca y producto & Postgrado & Al menos 2 años & $21.000 €$ bruto/año \\
\hline 2 & Community Manager & BCN & MK y Com - RRPP $y$ evertos & Lic. Publicidad y RR.PP. & Al menos 1 año & $12.000 € 15.000 €$ bruto/año \\
\hline 3 & ERECLUITER / COMMUNITY MANAGER & MD & Recur sos Humanos - Selec ción de Personal & Licenciado & Al menos 3 años & - \\
\hline 4 & Manager Presencia Online & MD & MK y Com - Desarrollo de marca y producto & Diplomado & Más de 5 años & - \\
\hline 5 F & Responsable MKT Online & MD & MK y Com - Comunic ación Corporativa & Lic. Comunicación Audiovisual & Al menos 3 años & - \\
\hline \begin{tabular}{|c|c|c|}
6 & \\
\end{tabular} & Online Specialist & MD & MK y Com - Desarrollo de marca y producto & Licenciado & Al menos 3 años & - \\
\hline 7 & Técnico Espeoialista E-Commerce & MD & MK y Com - Marketing & Lic. Publicidad y RR.PP. & De 3 a 5 años & - \\
\hline \begin{tabular}{|c|}
8 \\
\end{tabular} & Community Manager Freelance & MD & MK y Com - Marketing & F.S. - Comercio y Marketing & Al menos 1 año & - \\
\hline $9 \mathbf{S t}^{9}$ & Search Marketing Specialist & BCN & MK $y$ Com - Marketing & - & Al menos 2 años & - \\
\hline 10 & Community Manager & BCN & MK y Com - Marketing & - & No requerida & $18.000 € 21.000 €$ bruto/año \\
\hline 11 & Assistart Community Manager & MD & MK y Com - Desarrollo de marca y producto & Lic. Comunicación Audiovisual & No requerida & - \\
\hline 12 & Community Manager / Digital PR & MD & MK y Com - Comunic ación Corporativa & Lic. Periodismo & Al menos 1 año & $12.000 € 18.000 €$ bruto/año \\
\hline 13 & Especialista en Comunicación e Irternet & MD & MK y Com - Marketing & Licenciado & Al menos 3 años & - \\
\hline 14 & Periodista, Communit y Manager & Otra & MK y Com - Periodismo y edición & Lic. Comunicación Audiovisual & No requerida & - \\
\hline 15 & Community Manager & $B C N$ & MK y Com - Marketing & Bachillerato & Al menos 1 año & - \\
\hline 18 & Community Manager & BCN & MK y Com - RRPP y evertos & Lic. Publicidad y RR.PP. & No requerida & - \\
\hline 17 & Web Master - Community Manager & MD & INF y Teleco - programación & F.S. - Informática & No requerida & 450 € brutosimes \\
\hline 18 & Community Manager Junior & Otra & MK y Com - Desarrollo de marca y producto & Diplomado & Al menos 1 año & - \\
\hline 19 & Redactor de cortenidos. Community Manager & BCN & MKK y Com - Periodismo y edición & Lio. Periodismo & No requerida & - \\
\hline 20 & Community Manager & MD & MK $y$ Com - Marketing & Lic. Publicidad y RR.PP. & No requerida & - \\
\hline 21 & Community Manager & MD & MK y Com - Marketing & - & Al menos 1 año & $15.000 € 18.000 €$ bruto/año \\
\hline 22. & Junior Digital Marketing Manager & MD & MK y Com - Marketing & Lic. Adm. y Dirección Empresas & Al menos 2 años & $18.000 € 24.000 €$ bruto/año \\
\hline 23 & Community Manager sector automóvil & MD & Comercial y Vertas - Gran cuenta & Lic. Periodismo & Al menos 1 año & $21.000 € 24.000 €$ bruto/año \\
\hline 24 & Community Manager & Otra & MK y Com - Marketing & Licenciado & Al menos 2 años & - \\
\hline 20 & E-mail marketing - Community Manager & $B C N$ & Diseño y artes gráfic as - Diseño web & - & Al menos 2 años & - \\
\hline 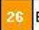 & Beca Pictures - Marketing Online & MD & MK y Com - Marketing & Cursando Licenciatura & No requerida & - \\
\hline
\end{tabular}

Cuadro 2. Ofertas de empleo con las palabras clave "Community Manager"

Como vemos en la tabla anterior, el $75 \%$ de todas estas ofertas están categorizadas dentro de la temática "Marketing y Comunicación", existiendo una mayor variedad en la subcategoría: desarrollo de marca y producto, RR.PP. y eventos, comunicación corporativa, marketing y periodismo y edición. El $58 \%$ de estas ofertas corresponden a la provincia de Madrid y el $31 \%$ a Barcelona.

El nivel de estudios requerido se caracteriza por la diversidad: Licenciado en Publicidad y RR.PP., Licenciado sin especificar la titulación y ningún nivel de estudios especificado son las tres categorías más numerosas, con cuatro casos cada una, seguidas de Licenciado en Periodismo, Licenciado en Comunicación Audiovisual y Diplomado, con tres casos cada una. Por tanto, podemos decir que, pese a que la temática principal en la que se organizan estas ofertas es "Marketing y Comunicación", el perfil del profesional del Community Manager, en lo que a su formación académica se refiere, no está todavía demasiado definido.

En cuanto a la experiencia mínima requerida, en la mayoría de ofertas (27\%) se indica "al menos 1 año", 6 ofertas especifican "no requerida", 5 ofertas señalan "al menos 2 años" y 4 ofertas solicitan "al menos 3 años". De esta manera, parece ser que, en general, se busca un perfil junior, con poca o ninguna experiencia en el sector, dada la actualidad de esta nueva profesión. Tan sólo 8 de las 26 ofertas listadas incluyen información sobre el salario, rondando la mayoría entre los $12.000 €$ y los $24.000 €$ brutos al año. 
El Community Manager es un profesional de la comunicación encargado de escuchar a la comunidad online, relacionarse con ella en nombre de la empresa, hacer llegar a su compañía lo que se dice de ella en el mundo digital para identificar amenazas u oportunidades e integrar estos espacios en las estrategias de negocio de la empresa. Todo ello con inmediatez y transparencia, utilizando un lenguaje cercano y ofreciendo contenidos relevantes.

En las plataformas sociales es fundamental monitorizar en tiempo real, escuchar activamente, participar en conversaciones con un tono próximo y cercano, ofrecer actualizaciones frecuentes con contenidos relevantes y de calidad, responder de manera rápida y transmitir transparencia y confianza, todas ellas tareas del Community Manager.

El auge de este nuevo perfil profesional hizo que en 2008 naciera la Asociación Española de Responsables de Comunidad Online (AERCO), una entidad sin ánimo de lucro compuesta por profesionales relacionados con las comunidades virtuales y que tiene la misión de representar y atender las necesidades de los responsables de comunidades online, con los objetivos de impulsar su desarrollo personal y profesional y lograr una proyección creciente y sostenible. AERCO ofrece cursos certificados de especialización para Community Managers.

Según el Libro Blanco "La función del Community Manager", publicado por AERCO junto con Territorio Creativo, "las organizaciones deberían empezar a comprender que la gestión de los medios sociales comienza a ser una función en sí misma, y replantearse su organización desde la base" (2009: 6). AERCO (2009: 5) define al Community Manager como:

aquella persona encargada/responsable de sostener, acrecentar y, en cierta forma, defender las relaciones de la empresa con sus clientes en el ámbito digital, gracias al conocimiento de las necesidades y los planteamientos estratégicos de la organización y los intereses de los clientes. Conoce los objetivos y actúa en consecuencia para conseguirlos. Muy genéricamente, podríamos decir que un Community Manager es aquella persona que preserva la identidad digital de la compañía.

\section{Resultados}

La amplia oferta de formación superior especializada en marketing digital e interactivo, especialmente en lo relacionado con el Social Media Marketing, existente en el curso académico 2010-2011 queda patente en un inicio al realizar una búsqueda en el buscador Google de las palabras clave "curso redes sociales" y "curso community manager"3.

La búsqueda de "curso redes sociales" genera más de 10 millones de resultados, mientras que "curso community manager" obtiene más de 600.000 resultados. En ambos casos, los resultados naturales están acompañados anuncios y enlaces patrocinados (Search Engine Marketing) en la parte superior y la columna lateral derecha, lo que demuestra el interés que generan las dos búsquedas para escuelas e instituciones con oferta formativa en la materia.

\footnotetext{
${ }^{3}$ Búsquedas llevadas a cabo el día 26 de abril de 2011.
} 


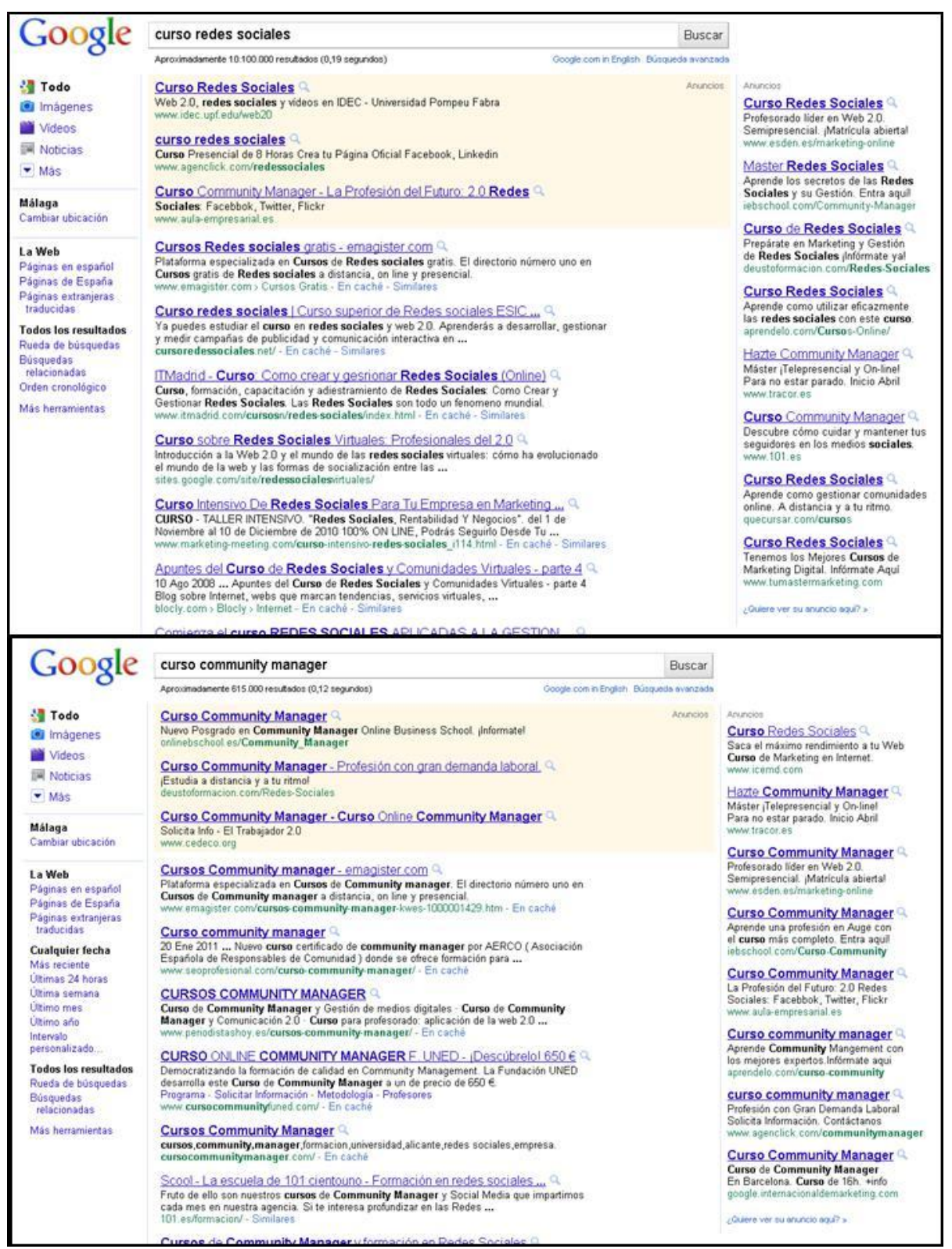

Cuadro 3. Resultados en Google de las palabras clave "curso redes sociales" y "curso community manager"

Una vez obtenidos estos resultados, se selecciona de manera aleatoria una muestra de cursos, postgrados y másters de formación superior en Marketing Digital y Community Management en el curso académico 2010-2011. Esta oferta formativa procede tanto de universidades públicas y privadas como de escuelas de negocios y asociaciones del sector. En la siguiente tabla se muestran algunos de los cursos obtenidos en la búsqueda: 


\begin{tabular}{|c|c|c|c|}
\hline & Institución & Estudios & Enlace \\
\hline 1 & Madrid School of Marketing & $\begin{array}{l}\text { Máster en Marketing Digital e } \\
\text { Interactivo }\end{array}$ & $\begin{array}{l}\text { http://Mww madridschoolofin arketing.es/Mas } \\
\text { ter_en_Marketing_Digital_Interactivo.htm }\end{array}$ \\
\hline 2 & $\begin{array}{l}\text { Universidad Internacional } \\
\text { de Cataluña }\end{array}$ & $\begin{array}{l}\text { Máster en proyectos de comunicación } \\
\text { online }\end{array}$ & http://www uic es/es/com unic ac ion-online \\
\hline 3 & ICEMD & $\begin{array}{l}\text { Máster Europeo en Marketing en } \\
\text { Internet y Publicidad Digital }\end{array}$ & $\begin{array}{l}\text { http: ///Www icemd.com/Program a-Superior- } \\
\text { de-M arketing-en-Internet-y-Publicidad- } \\
\text { Digital_ficha_6237_8322.htmI }\end{array}$ \\
\hline 4 & $\begin{array}{l}\text { Instituto de Empresa } \\
\text { Business School }\end{array}$ & Master in Digital Marketing & http://m aster-advertising. ie.edu/ \\
\hline 5 & Universidad de Barcelona & $\begin{array}{l}\text { Postgrado en Community Manager y } \\
\text { Social Media }\end{array}$ & $\begin{array}{l}\text { http: } / / \text { www wh ub.edu/web/ub/c a/es tudis/oferta } \\
\text { form ativa/masters_propis/fitxa/c/200911732/ } \\
\text { index.ntm। }\end{array}$ \\
\hline 6 & Fundesem & $\begin{array}{l}\text { Curso Superior de Community } \\
\text { Manager }\end{array}$ & $\begin{array}{l}\text { http } / / \text { www fundesem .es/curso_corto. asp?tip } \\
0=28 \text { curso }=80\end{array}$ \\
\hline 7 & ESUMA & $\begin{array}{l}\text { Executive PYME 2.0: EI Community } \\
\text { Manager en la empresa }\end{array}$ & $\begin{array}{l}\text { http: //Www esum a com/form acion/titulacione } \\
\text { s/executives/cm/ }\end{array}$ \\
\hline 8 & $\begin{array}{l}\text { Universidad Cardenal } \\
\text { Herrera-CEU }\end{array}$ & $\begin{array}{l}\text { Máster Oficial en Comunicación y } \\
\text { Branding Digital }\end{array}$ & $\begin{array}{l}\text { http://Www uchceu es/estudios/posgrado/ma } \\
\text { ster_universitario/comunic acion_digital/index } \\
\text { asp }\end{array}$ \\
\hline 9 & Escuela de Ejecutivos & $\begin{array}{l}\text { Curso de Marketing Online y Redes } \\
\text { Sociales }\end{array}$ & $\begin{array}{l}\text { http://Www .ede.com es/cursos/internet/m ark } \\
\text { eting-online-redes-sociales php }\end{array}$ \\
\hline 10 & AERCO & $\begin{array}{l}\text { Curso de especialización para } \\
\text { Community Manager }\end{array}$ & $\begin{array}{l}\text { http://www aercom unidad org/cursos- } \\
\text { com m unity-manager-form acion/ }\end{array}$ \\
\hline 11 & Universidad de Alicante & $\begin{array}{l}\text { Titulo de Experto Universitario en } \\
\text { Dirección y Gestión de redes sociales }\end{array}$ & $\begin{array}{l}\text { http://cursocom m unitym anager.com/experto } \\
\text { universitario/ }\end{array}$ \\
\hline 12 & $\begin{array}{l}\text { Universidad Camilo José } \\
\text { Cela }\end{array}$ & $\begin{array}{l}\text { Community Manager: gestión de } \\
\text { comunidades online }\end{array}$ & $\begin{array}{l}\text { http: //Www ucjc. edu/index.php?section= estu } \\
\text { dios/titulaciones/cursos-verano- } \\
11 / \text { com munity_manager }\end{array}$ \\
\hline 13 & IEB School & $\begin{array}{l}\text { Máster en Community Management: } \\
\text { empresa } 2.0 \text { y redes sociales }\end{array}$ & 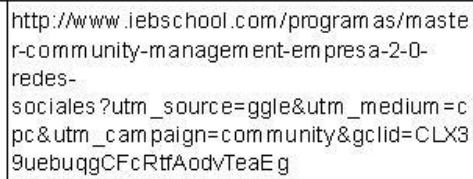 \\
\hline 14 & ESIC & $\begin{array}{l}\text { Máster Europeo en Marketing en } \\
\text { Internet y publicidad Digital }\end{array}$ & $\begin{array}{l}\text { http: //Www esic.es/ficha_titulacion/95/MMIP } \\
\text { D/master-europeo-en-marketing-en-intemet- } \\
\text { y-publicidad-digital }\end{array}$ \\
\hline
\end{tabular}

Cuadro 4. Oferta de formación superior en Marketing Digital y Community Management

Por otro lado, el análisis de los planes de estudio del Grado en Publicidad y RR.PP. en aquellas universidades en las que se ofrece, así como de otras titulaciones relacionadas en otras universidades en las que no se imparte este Grado (Marketing e Investigación de mercados o Comunicación), demuestra la segunda hipótesis, es decir, la escasa presencia de módulos y/o asignaturas en los planes de estudio del Grado en Publicidad y RR.PP. centradas en el estudio de la comunicación publicitaria online.

En las siguientes tablas se muestran los resultados obtenidos en el análisis de las 40 universidades de la muestra: 


\begin{tabular}{|c|c|c|c|c|c|}
\hline & Universidad & Grado & Asignaturas & Tipo & Curso \\
\hline 1 & Universidad de Almería & $\begin{array}{l}\text { Grado en Marketing e } \\
\text { Investigación de mercados }\end{array}$ & 1. Comercio electrónico & Optativa & Cuarto \\
\hline \multirow{2}{*}{2} & \multirow{2}{*}{ Universidad de Cádiz } & \multirow{2}{*}{ Grado en Publicidad y RR.PP. } & 1. Comunicación digital & Optativa & Cuarto \\
\hline & & & 2. Medios y soportes interactivos & Optativa & Cuarto \\
\hline 3 & Unive rsidad de Granada & $\begin{array}{l}\text { Grado en Marketing e } \\
\text { Investigación de mercados }\end{array}$ & - & - & - \\
\hline 4 & Unive rsidad de Málaga & Grado en Publicidad y RR.PP. & $\begin{array}{l}\text { 1. Creación de campañas en entornos } \\
\text { digitales }\end{array}$ & - & Cuarto \\
\hline 5 & Unive rsidad de Sevilla & Grado en Publicidad y RR.PP. & - & - & - \\
\hline 6 & Unive rsida d San Jorge & Grado en Publicidad y RR.PP. & 1. Marketing de guerrilla e Internet & Obligatoria & Cuarto \\
\hline 7 & $\begin{array}{l}\text { Universidad Europea Miguel } \\
\text { de Cervantes }\end{array}$ & Grado en Publicidad y RR.PP. & 1. Marketing en Internet & Optativa & - \\
\hline \multirow{2}{*}{8} & \multirow{2}{*}{ IE Universi dad } & \multirow{2}{*}{ Grado en Comunicación } & 1. Comunidades virtuales & Obligatoria & Tercero \\
\hline & & & 2. Comercio electrónico & Obligatoria & Tercero \\
\hline \multirow[b]{2}{*}{9} & \multirow{2}{*}{ Universidad de León } & \multirow{2}{*}{$\begin{array}{l}\text { Grado en Marketing e } \\
\text { Investigación de mercados }\end{array}$} & - & - & - \\
\hline & & & \multicolumn{3}{|c|}{$\begin{array}{l}\text { No disponible el plan de estudios de segundo, tercer y } \\
\text { cuarto curso }\end{array}$} \\
\hline \multirow{2}{*}{10} & \multirow{2}{*}{$\begin{array}{l}\text { Unive rsidad Pontificia de } \\
\text { Salamanca }\end{array}$} & \multirow{2}{*}{ Grado en Publicidad y RR.PP. } & 1. Publicidad interactiva & Obligatoria & Tercero \\
\hline & & & 2. Marketing online & Optativa & Cuarto \\
\hline \multirow{3}{*}{11} & \multirow{3}{*}{ Unive rsidad de Valladolid } & \multirow{3}{*}{ Grado en Publicidad y RR.PP. } & 1. Creatividad online & Optativa & Cuarto \\
\hline & & & 2. Redes sociales de comunicación & Optativa & Cuarto \\
\hline & & & 3. Publicidad interactiva & Optativa & Cuarto \\
\hline 12 & Unive rsidad Abat Oliba CEU & Grado en Publicidad y RR.PP. & - & - & - \\
\hline 13 & $\begin{array}{l}\text { Unive rsidad Autónoma de } \\
\text { Barcelona }\end{array}$ & Grado en Publicidad y RR.PP. & - & - & - \\
\hline \multirow{2}{*}{14} & \multirow{2}{*}{ Universidad de Barcelona } & \multirow{2}{*}{ Grado en Publicidad y RR.PP. } & - & - & - \\
\hline & & & \multicolumn{3}{|c|}{ No disponible el listado de optativas de cuarto curso } \\
\hline 15 & Universidad de Gerona & Grado en Publicidad y RR.PP. & $\begin{array}{l}\text { 1. Dentro del módulo "Comunicación } \\
\text { audiovisual e hipermedia" ( } 30 \text { créditos) } \\
\text { se incluye "Entornos de la Web } 2.0 \text { " }\end{array}$ & Obligatoria & Segundo \\
\hline \multirow{2}{*}{16} & \multirow{2}{*}{$\begin{array}{l}\text { Unive rsidad Internacional de } \\
\text { Cataluña }\end{array}$} & \multirow{2}{*}{ Grado en Publicidad y RR.PP. } & - & - & - \\
\hline & & & $\begin{array}{l}\text { No disponible el plan de estudios de cu } \\
\text { disponible el listado de optativas de tero }\end{array}$ & $\begin{array}{l}\text { uarto curso. } 1 \\
\text { rcer curso }\end{array}$ & No \\
\hline \multirow{2}{*}{17} & \multirow{2}{*}{ Universitat Oberta Catalunya } & \multirow{2}{*}{ Grado en Comunicación } & - & - & - \\
\hline & & & \multicolumn{3}{|c|}{ No disponible el listado de optativas de cuarto curso } \\
\hline 18 & Unive rsidad Pompeu Fabra & Grado en Publicidad y RR.PP. & $\begin{array}{l}\text { 1. Análisis de mensajes en medios } \\
\text { interactivos }\end{array}$ & Obligatoria & Segundo \\
\hline \multirow{2}{*}{19} & \multirow{2}{*}{ Universidad Ramón Llull } & \multirow{2}{*}{ Grado en Publicidad y RR.PP. } & - & - & - \\
\hline & & & \multicolumn{3}{|c|}{ No disponible el listado de optativas de cuarto curso } \\
\hline 20 & Unive rsitat Rovira i Virgili & Grado en Publicidad y RR.PP. & 1. Marketing directo e interactivo & Optativa & Cuarto \\
\hline
\end{tabular}

Cuadro 5. Las asignaturas de comunicación publicitaria online en el Grado 


\begin{tabular}{|c|c|c|c|c|c|}
\hline & Universidad & Grado & Asignaturas & Tipo & Curso \\
\hline \multirow{2}{*}{21} & \multirow{2}{*}{ Universidad de Vic } & \multirow{2}{*}{ Grado en Publicidad y RR.PP. } & 1. Comunicación digital interactiva & Obligatoria & Tercero \\
\hline & & & \multicolumn{3}{|c|}{ No disponible el listado de optativas de tercer y cuarto curso } \\
\hline \multirow{2}{*}{22} & \multirow{2}{*}{$\begin{array}{l}\text { Universidad Alfonso } X \text { EI } \\
\text { Sabio }\end{array}$} & \multirow{2}{*}{ Grado en Comunicación } & - & - & - \\
\hline & & & \multicolumn{3}{|c|}{ No disponible el listado de optativas de cuarto curso } \\
\hline 23 & Universidad Nebrija & Grado en Publicidad & 1. Publicidad interactiva & Obligatoria & Segundo \\
\hline 24 & $\begin{array}{l}\text { Universidad Camilo José } \\
\text { Cela }\end{array}$ & Grado en Publicidad y RR.PP. & 1. Comunicación en entornos digitales & Obligatoria & Tercero \\
\hline 25 & $\begin{array}{l}\text { Universidad Complutense de } \\
\text { Madrid }\end{array}$ & Grado en Publicidad y RR.PP & - & - & - \\
\hline \multirow{5}{*}{26} & \multirow{5}{*}{$\begin{array}{l}\text { Universidad Europea de } \\
\text { Madrid }\end{array}$} & \multirow{5}{*}{$\begin{array}{l}\text { Grado en Comunicación } \\
\text { Publicitaria }\end{array}$} & 1. Digital advertising & - & Primero \\
\hline & & & 2. Publicidad en entornos digitales & - & Segundo \\
\hline & & & $\begin{array}{l}\text { 3. Redes sociales y nuevas } \\
\text { tendencias }\end{array}$ & - & Segundo \\
\hline & & & 4. Comercio electrónico & - & Cuarto \\
\hline & & & 5. Estrategia digital e interactiva & - & Cuarto \\
\hline \multirow{2}{*}{27} & \multirow{2}{*}{$\begin{array}{l}\text { Universidad Francisco de } \\
\text { Vitoria }\end{array}$} & \multirow{2}{*}{ Grado en Publicidad } & $\begin{array}{l}\text { 1. Publicidad interactiva y nuevos } \\
\text { soportes }\end{array}$ & Optativa & Tercero \\
\hline & & & 2. Taller de publicidad digital & Optativa & Tercero \\
\hline \multirow{2}{*}{28} & \multirow{2}{*}{ Universidad Rey Juan Carlos } & \multirow{2}{*}{ Grado en Publicidad y RR.PP } & 1. Comunicación multimedia & Obligatoria & Tercero \\
\hline & & & \multicolumn{3}{|c|}{ No disponible el listado de optativas de cuarto curso } \\
\hline 29 & Universidad San Pablo CEU & Grado en Publicidad y RR.PP & - & - & - \\
\hline \multirow{2}{*}{30} & \multirow{2}{*}{ Universidad de Navarra } & \multirow{2}{*}{ Grado en Publicidad y RR.PP. } & 1. Comunicación multimedia & - & Primero \\
\hline & & & \multicolumn{3}{|c|}{ No disponible el listado de optativas de tercer y cuarto curso } \\
\hline 31 & Universidad de Alicante & Grado en Publicidad y RR.PP & - & - & - \\
\hline 32 & Universidad Jaime I & Grado en Publicidad y RR.PP & 1. Marketing directo e interactivo & Optativa & Cuarto \\
\hline 33 & $\begin{array}{l}\text { Universidad Cardenal } \\
\text { Herrera - CEU }\end{array}$ & Grado en Publicidad y RR.PP & - & - & - \\
\hline 34 & Universidad de Vigo & Grado en Publicidad y RR.PP & $\begin{array}{l}\text { 1. Comunicación alternativa: medios } \\
\text { virtuales y nuevos soportes }\end{array}$ & Optativa & Cuarto \\
\hline \multirow{2}{*}{35} & \multirow{2}{*}{ Universidad de Deusto } & \multirow{2}{*}{ Grado en Comunicación } & - & - & - \\
\hline & & & \multicolumn{3}{|c|}{ No disponible el listado de optativas de cuarto curso } \\
\hline 36 & Universidad del País Vasco & Grado en Publicidad y RR.PP & 1. Marketing estratégico e interactivo & Obligatoria & Cuarto \\
\hline \multirow{2}{*}{37} & \multirow{2}{*}{ Universidad de Oviedo } & \multirow{2}{*}{$\begin{array}{l}\text { Grado en Comercio y } \\
\text { Marketing }\end{array}$} & - & - & - \\
\hline & & & \multicolumn{3}{|c|}{$\begin{array}{l}\text { No disponible el plan de estudios de segundo, tercer y } \\
\text { cuarto curso }\end{array}$} \\
\hline 38 & $\begin{array}{l}\text { Universidad Católica San } \\
\text { Antonio }\end{array}$ & Grado en Publicidad y RR.PP & 1. Publicidad interactiva & Optativa & - \\
\hline 39 & Universidad de Murcia & Grado en Publicidad y RR.PP & $\begin{array}{l}\text { 1. Nuevas tendencias en } \\
\text { comunicación publicitaria }\end{array}$ & Optativa & Cuarto \\
\hline 40 & ESIC Business Marketing & I & 1. Comunicación multimedia & - & Tercero \\
\hline 140 & School & 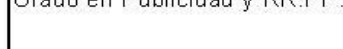 & 2. Publicidad interactiva & - & Cuarto \\
\hline
\end{tabular}

Cuadro 6. Las asignaturas de comunicación publicitaria online en el Grado 
En las 40 universidades analizadas se han encontrado 35 asignaturas cuya denominación entronca con el marketing y la comunicación publicitaria online, lo que supone una media de 0,88 por Universidad. Por lo tanto, no todos los Grados en Publicidad y RR.PP. analizados cuentan con una asignatura específica en este ámbito.

En total, de las 40 universidades analizadas, son $24(60 \%)$ las que tienen asignaturas, de tal manera que la media de asignaturas para las universidades con oferta formativa en esta temática es de 1,35 asignaturas. De estas 24 universidades, 17 cuentan con una asignatura en este ámbito $(71 \%), 5$ tienen dos asignaturas $(21 \%)$ y una Universidad $(12,5 \%)$ tiene tres asignaturas (Universidad de Valladolid). Tan sólo la Universidad Europea de Madrid ofrece más de tres asignaturas, al incluir cinco en el plan de estudios del Grado en Publicidad y RR.PP.

Cabe matizar que de las 16 universidades que no ofrecen en los planes de estudio del Grado en Publicidad y RR.PP. asignaturas de marketing y/o comunicación publicitaria online (el 40\% de la muestra), 8 universidades no dan información completa, al no aparecer en varios casos el plan de estudios de algún curso ni las optativas en otros, por lo que puede que sí incluyan las asignaturas objeto de este estudio. Además, de las 24 universidades con asignaturas, tres no incluyen información sobre las optativas de tercer y/o cuarto curso, de tal manera que también existe la posibilidad de que cuenten con más oferta formativa en este temática.

Esta circunstancia se debe, en ambos casos, a la progresiva implantación de los nuevos estudios del Grado en Publicidad y RR.PP., ya que varias son las universidades que han optado por ir actualizando la información conforme se vaya implantando el Grado en cursos venideros (en el curso académico 2010-2011 todas las universidades ofrecen el primer curso del Grado, puesto que era el último año marcado para su implantación, según el plan de Bolonia).

El Grado en Publicidad y RR.PP. tiene un peso en la muestra analizada del $77,5 \%$, mientras que aquellas universidades que no contaban con esta titulación suponen el 22,5\%: cuatro universidades tienen el Grado en Marketing e Investigación de mercados (10\%) y cinco universidades cuentan con Grado en Comunicación (12,5\%). En este último caso, se incluye el Grado en Comunicación Publicitaria de la Universidad Europea de Madrid. Incluimos en la siguiente tabla el reparto de las universidades según el Grado analizado, tanto para la muestra total, como para las que tienen asignaturas de la temática estudiada y las que no. En las dos últimas columnas aparece el reparto de asignaturas según titulación (en absoluto y porcentaje): 


\begin{tabular}{|l|c|c|c|c|c|c|c|c|}
\cline { 2 - 10 } \multicolumn{1}{c|}{} & \multicolumn{2}{c|}{ Total muestra } & \multicolumn{2}{c|}{ Sin asignaturas } & \multicolumn{2}{c|}{ Con asignaturas } & \multicolumn{2}{c|}{$N^{\circ}$ de asignaturas } \\
\hline Grado en Publicidad y RR.PP. & 31 & $77,5 \%$ & 10 & $62,5 \%$ & 21 & $87,5 \%$ & 27 & $77,1 \%$ \\
\hline Grado en Marketing en Investigación de mercados & 4 & $10,0 \%$ & 3 & $18,8 \%$ & 1 & $\mathbf{4 , 2} \%$ & 1 & $2,9 \%$ \\
\hline Grado en Comunicación & 5 & $12,5 \%$ & 3 & $18,8 \%$ & 2 & $8,3 \%$ & 7 & $20,0 \%$ \\
\hline
\end{tabular}

Cuadro 7. Asignaturas en los Grados analizados

Las diez universidades que no tienen asignaturas de marketing y comunicación publicitaria online en el Grado en Publicidad y RR.PP. suponen el 32\% de las 31 universidades analizadas, mientras que las 21 universidades con asignaturas representan el 68\%. En el caso del Grado en Marketing e Investigación de mercados, las universidades que no tienen asignaturas son el $75 \%$ del total de esta titulación, mientras que para el Grado en Comunicación, 3 cuentan con asignaturas (60\%) y 2 no (40\%).

De media, los Grados en Publicidad y RR.PP. estudiados tienen 1,28 asignaturas de marketing y comunicación publicitaria online, al haber 27 asignaturas en 21 titulaciones. En los Grados en Comunicación, esta media asciende a 3,5 asignaturas para cada titulación, aunque ello se debe al sesgo que supone el Grado en Comunicación Publicitaria de la Universidad Europea de Madrid, con 5 de las 7 asignaturas de esta titulación.

Por lo que respecta al reparto por cursos, en la mayoría de casos (49\%) las asignaturas se ubican en el último curso de la titulación, nueve asignaturas en tercer curso $(26 \%)$, cinco en segundo curso $(14 \%)$ y dos en primer curso (5\%). Dos universidades no especifican el curso en el que se enmarcan las asignaturas de marketing y comunicación publicitaria online que ofrecen. Se trata de la Universidad Europea Miguel de Cervantes y la Universidad Católica San Antonio, en ambos casos en el Grado en Publicidad y RR.PP., con las asignaturas "Marketing en Internet" y "Publicidad interactiva", respectivamente. Ello se debe a que en ambos casos son asignaturas optativas, por lo que probablemente podrán cursarse en diferentes cursos.

En general, son los dos últimos cursos de la titulación los que cuentan con una mayor oferta de asignaturas de especialización, como es el caso de marketing y comunicación publicitaria online, ya que los dos primeros cursos incluyen asignaturas de formación básica, sugeridos por el Libro Blanco de ANECA y en ocasiones comunes entre los diferentes Grados en Comunicación (Publicidad y RR.PP., Comunicación Audiovisual y Periodismo), de tal manera que las propuestas formativas de cada Universidad se incluyen en el plan de estudios de tercer y/o cuarto curso. El perfil genérico de las asignaturas específicas de marketing y comunicación publicitaria online es, por tanto, optativas de cuarto curso. 
De hecho, 15 de las 35 asignaturas detectadas son optativas (43\%), 11 son obligatorias (31\%) y en nueve casos no se especifica (26\%). De las 15 asignaturas optativas, 11 pertenecen a cuarto curso (73\%) y representan el $65 \%$ de las asignaturas de asignaturas de cuarto curso.

Por último, hay varias asignaturas que aparecen con la misma denominación en más de un caso: por ejemplo, "Comercio electrónico" y "Comunicación multimedia", ambas en tres universidades y "Marketing digital e interactivo", en dos casos. Dos universidades definen asignaturas empleando las redes sociales: "Redes sociales de comunicación" (Universidad de Valladolid) y "Redes sociales y nuevas tendencias" (Universidad Europea de Madrid). La asignatura que más se repite es "Publicidad interactiva", en siete ocasiones, es decir, el 20\% de las asignaturas detectadas (incluyendo aquí también "Publicidad en entornos digitales").

\section{Conclusiones}

Llegados a este punto, podemos verificar la primera hipótesis de partida, la amplia oferta de formación superior (posgrados y másters) especializada en marketing digital e interactivo existente en el curso académico 2010-2011. Las enseñanzas de Máster tienen como finalidad la adquisición por parte del estudiante de una formación avanzada, de carácter especializado o multidisciplinar, orientada a la especialización académica o profesional, o bien a promover la iniciación en tareas investigadoras.

Al tratarse la comunicación publicitaria online de un ámbito de especialización dentro del amplio abanico de perfiles profesionales derivados del estudio del Grado en Publicidad y RR.PP., los contenidos relacionados con esta temática incluidos dentro de la titulación se caracteriza por su optatividad y su inserción principalmente en el último curso de la titulación, dado su carácter complementario.

Aunque el objeto de este estudio era analizar la existencia de asignaturas ad hoc para la comunicación publicitaria online en los planes de estudio del Grado en Publicidad y RR.PP., hay que tener en cuenta que, como medio publicitario convencional, la comunicación corporativa y publicitaria en Internet también será estudiada desde el punto de vista de la estrategia y la planificación dentro de los contenidos de otras materias de la titulación, como por ejemplo "Procesos y técnicas de la investigación, planificación y compra de medios y soportes" y "Fundamentos y técnicas de la estrategia de la comunicación (especialmente publicitaria y de las relaciones públicas", ambos contenidos comunes obligatorios (ANECA, 2005: 336).

En cuanto la segunda hipótesis, se demuestra la escasa presencia de módulos y/o asignaturas en los planes de estudio del Grado en Publicidad y RR.PP. centradas en el estudio de la comunicación publicitaria online, aunque con matices, ya que el $60 \%$ de los Grados sí tienen asignaturas, un porcentaje aunque no del $100 \%$ sí importante. En cualquier caso, parece ser que la inclusión de asignaturas de esta temática va a ser progresiva, conforme se 
vayan implantando los cursos, como muestra el hecho de que en varias universidades la información sobre el plan de estudios no está completa.

El desarrollo imparable de las acciones de publicidad interactiva y la explosión del Social Media Marketing obligarán a una revisión continua por parte de las universidades de las tendencias en el mercado laboral para ir adaptando la oferta de contenidos optativos de la titulación y así ofrecer a los alumnos una formación basada en competencias que les capaciten como profesionales y ayuden a su inserción en el ámbito profesional.

\section{Anexo}

Enlaces a las páginas web con los planes de estudio de los Grados en Publicidad y RR.PP. de la muestra estudiada:

1. Universidad de Almería - Grado en Marketing e Investigación de mercados: http://cms.ual.es/UAL/estudios/grados/GRAD06710

2. Universidad de Cádiz - Grado en Publicidad y RR.PP.: http://www.uca.es/es/tratarAplicacionAsignaturasPlanEstudios.do?idTitulacion=31309\&acces $\mathrm{o}=\mathrm{t}$

3. Universidad de Granada - Grado en Marketing e Investigación de mercados: http://grados.ugr.es/marketing/

4. Universidad de Málaga - Grado en Publicidad y RR.PP.:

http://www.cccom.uma.es/descargas/graduados/AdscripcionAsignaturasPUBRRPP.pdf

5. Universidad de Sevilla - Grado en Publicidad y RR.PP.:

http://www.us.es/estudios/grados/plan_211

6. Universidad San Jorge - Grado en Publicidad y RR.PP.:

http://www.usj.es/estudios/oficiales/grados/publicidad

7. Universidad Europea Miguel de Cervantes - Grado en Publicidad y RR.PP.: http://www.uemc.edu/es/Estudios/Grados/Publicidad/Paginas/GradoenPublicidadyRelaciones Publicas.aspx

8. IE Universidad - Grado en Comunicación:

http://www.ie.edu/es/universidad/estudios/oferta-academica/grado-comunicacion

9. Universidad de León - Grado en Marketing e Investigación de mercados:

http://www.unileon.es/estudiantes/estudiantes-grado/oferta-de-estudios/grado-enmarketing-e-investigacion-de-mercados

10. Universidad Pontificia de Salamanca - Grado en Publicidad y RR.PP.: 
http://www.upsa.es/estudios/grados/publicidad/ficha.php

11. Universidad de Valladolid - Grado en Publicidad y RR.PP.:

http://www.uva.es/consultas/asignaturas.php?codigo_plan=458\&ano_academico=1011

12. Universidad Abat Oliba CEU - Grado en Publicidad y RR.PP.:

http://www.uao.es/es/estudios/grados/publicidad-y-rrpp

13. Universidad Autónoma de Barcelona - Grado en Publicidad y RR.PP.:

http://www.uab.es/servlet/Satellite/estudiar/todos-los-estudios/informacion-

general/publicidad-y-relaciones-publicas-grado-eees-

1099409747826. html?param1 $=1265367068074$ \&param10 $=4$

14. Universidad de Barcelona - Grado en Publicidad y RR.PP.:

http://www.ub.edu/web/ub/ca/estudis/oferta_formativa/graus/fitxa/P/G1054/index.html

15. Universidad de Gerona - Grado en Publicidad y RR.PP.:

http://www.udg.edu/tabid/10104/Default.aspx?ID=3108G0208\&language=ca-ES

16. Universidad Internacional de Cataluña - Grado en Publicidad y RR.PP.:

http://www.uic.es/ca/publicitat

17. Universitat Oberta de Catalunya - Grado en Comunicación:

http://www.uoc.edu/estudios/grados/comunicacion/presentacion/index.html

18. Universidad Pompeu Fabra - Grado en Publicidad y RR.PP.:

http://www.upf.edu/estudiants/es/titulacions/grau-publicitat/presentacio/index.html

19. Universidad Ramón Llull - Grado en Publicidad y RR.PP.:

http://www.blanquerna.url.edu/web/interior. aspx?alias=fcc.estudis-grau.publicitat-relacionspubliques\&lang=_es

20. Universitat Rovira i Virgili - Grado en Publicidad y RR.PP.:

http://www.urv.cat/cae/graus/es_graudepublicitat.html

21. Universidad de Vic - Grado en Publicidad y RR.PP.:

http://www.uvic.es/estudi/72

22. Universidad Alfonso X El Sabio - Grado en Comunicación:

http://www.uax.es/uax/que-estudiar/grado/comk/gco0

23. Universidad Nebrija - Grado en Publicidad:

http://www.nebrija.com/carreras-universitarias/publicidad-relaciones-publicas/index.htm

24. Universidad Camilo José Cela - Grado en Publicidad y RR.PP.: 
http://www.ucjc.edu/index.php?section=estudios/titulaciones/titulacionesoficiales/titulaciones-grado/publicidad-relaciones-publicas

25. Universidad Complutense de Madrid - Grado en Publicidad y RR.PP.: http://www.ucm.es/pags.php?a=estudios\&d=muestragrado\&idgr $=66$

26. Universidad Europea de Madrid - Grado en comunicación publicitaria: http://www.uem.es/titulacion/grado-en-comunicacion-publicitaria

27. Universidad Francisco de Vitoria - Grado en Publicidad: http://www.ufv.es/oferta-formativa/grado-en-publicidad_1417

28. Universidad Rey Juan Carlos - Grado en Publicidad y RR.PP.: http://www.urjc.es/estudios/grado/publicidad_rrpp/publicidad_rrpp.html 29. Universidad San Pablo CEU - Grado en Publicidad y RR.PP.: http://www.uspceu.com/pdf/oferta-academica/2011-12/HUMANIDADES-6.pdf 30. Universidad de Navarra - Grado en Publicidad y RR.PP.: http://www.unav.es/fcom/programas/grado/publicidad/gradopub.htm 31. Universidad de Alicante - Grado en Publicidad y RR.PP.: http://cv1.cpd.ua.es/webcvnet/planestudio/planestudiond.aspx?plan=C154

32. Universidad Jaime I - Grado en Publicidad y RR.PP.: http://www.uji.es/infoest/estudis/grau/esp/csj/publi.html

33. Universidad Cardenal Herrera CEU - Grado en Publicidad y RR.PP.: http://www.uchceu.es/estudios/grado/publicidad_relaciones_publicas.aspx

34. Universidad de Vigo - Grado en Publicidad y RR.PP. http://webs.uvigo.es/victce/index.php?option=com_content\&task=view\&id=1527\&Itemid=6 08

35. Universidad de Deusto - Grado en Comunicación: http://www.deusto.es/servlet/Satellite/Estudio/1246882274545/_cast/\%231102609955027 \%231233584563466\%231242048302768/0/c0/UniversidadDeusto/comun/render?tipoColecc ion=Page

36. Universidad del País Vasco - Grado en Publicidad y RR.PP.: http://www.ehu.es/p200content/es/contenidos/grado/act_gr_gpubli30_323/es_esgpu/es_filegr_gpubli30_323.html 37. Universidad de Oviedo - Grado en Comercio y Marketing: 
http://www.uniovi.es/descubreuo/ofertaformativa/grados/-

/asset_publisher/Zt3Q/content/grado-en-comercio-y-

marketing?redirect $=\% 2$ Fdescubreuo $\% 2$ Fofertaformativa $\% 2$ Fgrados

38. Universidad Católica San Antonio - Grado en Publicidad y RR.PP.:

http://www.ucam.edu/estudios/grados/publicidad

39. Universidad de Murcia - Grado en Publicidad y RR.PP.:

https://suma.um.es/suma/servlet/sumav2.general.Suma?oper=administrativa/visualizaGdoc enteGrado. intersticial\&codTitu=227\&codCen $=0$ E39\&cursoAca $=2010$ \&pagina $=$ visualizaPresen tacion

40. ESIC Business Marketing School - Grado en Publicidad y RR.PP.:

http://www.esic.es/ficha_titulacion/63/e/grado-oficial-publicidad-y-relaciones publicasmadrid

\section{REFERENCIAS BIBLIOGRÁFICAS}

ACED, Cristina (2010): Perfiles profesionales 2.0. Barcelona, Editorial OUC.

Asociación De Responsables De Comunidad Online (2009): La función del community manager. Madrid, AERCO, en

http://www.box.net/shared/pgur4btexi (consultado 30/04/2011).

Asociación Para la Investigación De Medios De Comunicación (AIMC) (2011): Estudio general de medios. Madrid, AIMC, en http://www.aimc.es/-Audiencia-deInternet-en-el-EGM-.html (consultado 30/04/2011).

Asociación Para la investigación de Medios de Comunicación (AimC) (2011): Navegantes en la Red. Madrid, AIMC, en http://www.aimc.es/-Navegantesen-la-Red-.html (consultado 30/04/2011).

AnecA (2005): Libro Blanco. Títulos de grado en Comunicación. Madrid, Agencia Nacional de Evaluación de la Calidad y Acreditación, en http://www.aneca.es/var/media/150336/libroblanco_comunicacion_def.pdf (consultado 13/04/2011).

Benavides Delgado, Juan et al. (2008): Nuevas tendencias de la comunicación. Madrid, Universidad Complutense de Madrid. 
Bermejo BerRos, Jesús (2008): "El receptor publicitario del siglo XXI en el marco de la interactividad: entre el consumer y el prossumer", en Pacheco, M. (co.), La publicidad en el contexto digital. Sevilla, Comunicación Social.

CASTelló MartíneZ, Araceli (2010): Estrategias empresariales en la Web 2.0. Las redes sociales online. Alicante, ECU.

CAMPOS FREIRE, Francisco (2008): "Las redes sociales trastocan los modelos de los medios de comunicación tradicionales", en Revista Latina de Comunicación Social, no 63, pp. 287-293, en http://www.ull.es/publicaciones/latina/_2008/23_34_Santiago/Francisco_Ca mpos.html (consultado: 19/01/2011).

CelayA, Javier (2008): La empresa en la Web 2.0. Madrid, Gestión 2000.

Fanjul PeYró, Carlos y GOnZÁlez oñATE, Cristina (2010): "Nuevas metodologías docentes para el grado de Publicidad y Relaciones Públicas de la Universidad Jaume I de Castellón", en Sierra Sánchez, J. y Sotelo González, J., Métodos de innovación docente aplicados a los estudios de Ciencias de la Comunicación. Madrid, Fragua, pp. 318-332.

INFOADEX (2011): Estudio InfoAdex de la inversión publicitaria en España. Madrid, InfoAdex, en http://www.infoadex.es/estudios.html\# (consultado 28/04/2011).

INTERACTIVe AdVERTISINg BuREAu SPAin (2010): Tendencias del mercado laboral en el sector de la publicidad digital. Madrid, IAB Spain, en http://www.iabspain.net/ver.php?identificador=64\&mod=noticias (consultado 15/04/2011).

MAdinABeitiA, Eduardo (2010): "La publicidad en medios interactivos. En busca de nuevas estrategias", en Telos, no 82, pp. 43-54, en http://sociedadinformacion. fundacion.telefonica.com/seccion=1268\&idioma= es_ES\&id=2010020211580001\&activo=6.do (consultado: 20/01/2011).

Marta Lazo, Carmen (2010): El EEeS y el Proyecto Final en los Grados de Comunicación. Madrid, Fragua. 
Meso, Koldo, et al. (2010): "Evolución de la docencia universitaria: utilización académica de las redes sociales dentro del marco del EEES", en Actas II Congreso Internacional Latina de Comunicación Social, La Laguna, en http://www.revistalatinacs.org/10SLCS/actas_2010/036_Meso.pdf (consultado: 26/04/2011).

Pérez Serrano, María José, et al. (2010): "El sistema 'ECTS' y el mapa de competencias como elementos vertebradores de los nuevos estudios de grado en comunicación", en Quaderns Digitals, no 61, en http://www.quadernsdigitals.net/index.php?accionMenu=hemeroteca.Visuali zaArticuloIU.visualiza\&articulo_id=10917 (consultado: 28/04/2011).

ReAl DeCRETo 1393/2007, de 29 de octubre, por el que se establece la ordenación de las enseñanzas universitarias oficiales. Boletín Oficial del Estado, 30 de octubre de 2007, núm. 260, p. 44037.

Ros Diego, Vicente (2008): eBranding. Posiciona tu marca en la Red. A Coruña, NetBiblo.

Sierra Sánchez, Javier y CabezUelo Lorenzo, Francisco (2010): "Recursos de las facultades de comunicación para la formación en competencias en el marco del Espacio Europeo de Educación Superior", en Doxa Comunicación, no 11, pp. 31-54, en

http://www.humanidades.uspceu.es/pdf/RecursosdeFacultadesdecomunicaci onparaformacionEEES.pdf (consultado: 23/04/2011).

TERritorio Creativo (2010): Marketing en medios sociales, en http://www.territoriocreativo.es/etc/2010/04/estudio-social-mediaespana.html (consultado 21/04/2011).

UnIVERSAL MCCANN (2010): Wave 5. The socialisation of brands, en http://www.slideshare.net/Olivier.mermet/universal-mccann-wave-5-thesocialisation-of-brands (consultado 18/04/2011).

[Recibido: 3 de mayo de 2011. Aceptado (con solicitud de cambios): 24 de febrero de 2012. Recepción del artículo corregido: 25 de febrero de 2012]. 